\title{
Numerical Model for Human Induced Vibrations
}

\author{
Marcello Vanali ${ }^{1}$, Marta Berardengo ${ }^{1}$, Stefano Manzoni ${ }^{2}$ \\ 1 Dipartimento di Ingegneria Industriale, Università degli studi di Parma \\ 2 Dipartimento di Meccanica, Politecnico di Milano
}

\begin{abstract}
This paper proposes a new model for describing the effect of people on the dynamic behaviour of slender structures. The influence of people is described by using the so-called ground reaction forces. These are split into two types: passive ground reaction forces and active ground reaction forces. This enables to model the influence of both passive and active people occupying the structure.
\end{abstract}

The experimental tests carried out on two different test-case structures allowed to validate the proposed approach, proving its reliability as well as its accuracy.

KEYWORDS: Human structure interaction; ground reaction force; dynamics; vibration; slender structure

1 Introduction

The dynamic behaviour of structures occupied by people is studied since a lot of time. Particular attention has been devoted to the vibration serviceability issues of civil structures, with special focus on human induced vibrations and HumanStructure Interaction (HSI) [1][2][3][4][5]. Staircases [6][7], footbridges [8][9][10][11][12][13][14], and more generally pedestrian structures [15][16][17], have been deeply investigated and many other research works focus on stadia serviceability issues [18][19][20]. Furthermore, international standards and codes [21][22][23][24][25] have been developed to the aim of both designing and evaluating the structure dynamics under the crowd action and they are the natural reference when vibration serviceability is assessed. Unlike other standards, the recent guidance [26] (Joint Working Group, 2008) regarding dynamic performance requirements for permanent grandstands subjected to crowd action recommends to consider human structure interaction.

Many works proposed models to describe/predict the HSI effects on the structure dynamics (e.g., [9][15][16]). This work proposes a model where the action of each single person on the structure is taken into account and the structure is considered as a multi-degrees-of-freedom system. Therefore, even structures with coupled modes can be considered when computing the structural response due to people walking. 
The paper is structured as follows: Section 2 describes the theoretical approach and explains how to use the model proposed to predict structural vibrations in presence of people; Section 3 describes the experimental tests carried out for model validation.

\section{Theoretical model}

When a person is in contact with a point of a structure, he/she produces a Ground Reaction Force (GRF). The GRF is the total force exchanged between the person and the structure. The GRF can be split into passive GRF (PGRF) and active GRF (AGRF) [7]. The PGRF is a force generated by structural movement; when an external force acts on the structure, it vibrates and excites the person. If the person is considered as a dynamic system, it starts to vibrate as well. Therefore, a force is exerted by the person on the structure. Such a force is named PGRF. The AGRF is due to the active force generated by the person's active movement. The AGRFs do not depend on (and are not generated by) the vibration of the structure behind the person and are only due to the active movement of the person. The AGRF can be described as the force exerted by a moving person on a structure with an infinite stiffness.

To sum up, the PGRF depends on the dynamic features of the person and on the motion of the structure. Conversely, the AGRF is produced by an active movement which is not related to the dynamics of the structure.

According to Figure 1, the following equation describes the dynamics of the structure occupied by people:

$$
\mathbf{x}(\omega)=\mathbf{G}(\omega)\left(-\mathbf{f}^{\mathrm{ACTIVE}}(\omega)-\mathbf{f}^{\mathrm{GR}}(\omega)+\mathbf{f}(\omega)\right)
$$

Where $\mathbf{x}$ is the vector of the degrees-of-freedom in which the structure has been discretised, $\mathbf{G}$ is the matrix containing the frequency response functions (FRF) of the empty structure, $\mathbf{f}$ is the vector of the external forces, $\mathbf{f}^{\mathrm{ACTIVE}}$ is the vector of the AGRFs and $\mathbf{f}^{\mathrm{GR}}$ is the vector of the PGRFs. The expression of $\mathbf{G}$ is [27][28]:

$$
\mathbf{G}(\omega)=\sum_{j=1}^{n} \frac{\boldsymbol{\phi}_{j} \boldsymbol{\phi}_{j}^{\mathrm{T}}}{\omega_{j}^{2}-\omega^{2}+2 \mathrm{i} \zeta_{j} \omega \omega_{\mathrm{j}}}
$$

where $\omega_{\mathrm{j}}$ is the jth eigenfrequency, $\zeta_{j}$ is the associated non-dimensional damping ratio and $\boldsymbol{\phi}_{j}$ is the jth mode shape vector (scaled to the unit modal mass) measured/evaluated at discrete points (the superscript $T$ indicates transposition). Finally, $n$ is the (arbitrary) number of considered modes, $\omega$ is the circular frequency and i is the imaginary unit. Since the eigenvectors are known at discrete $(m)$ points, the matrix $\mathbf{G}(\omega)$ is the $m \times m$ matrix containing the FRFs for these points.

We can now take into account two different situations:

1. passive people (i.e., people who is not moving) on the structure;

2. active people (i.e., people who is moving) on the structure.

The two following subsections will show how to use the model of Eq. 1 to describe the dynamics of the whole system in the two listed cases. 


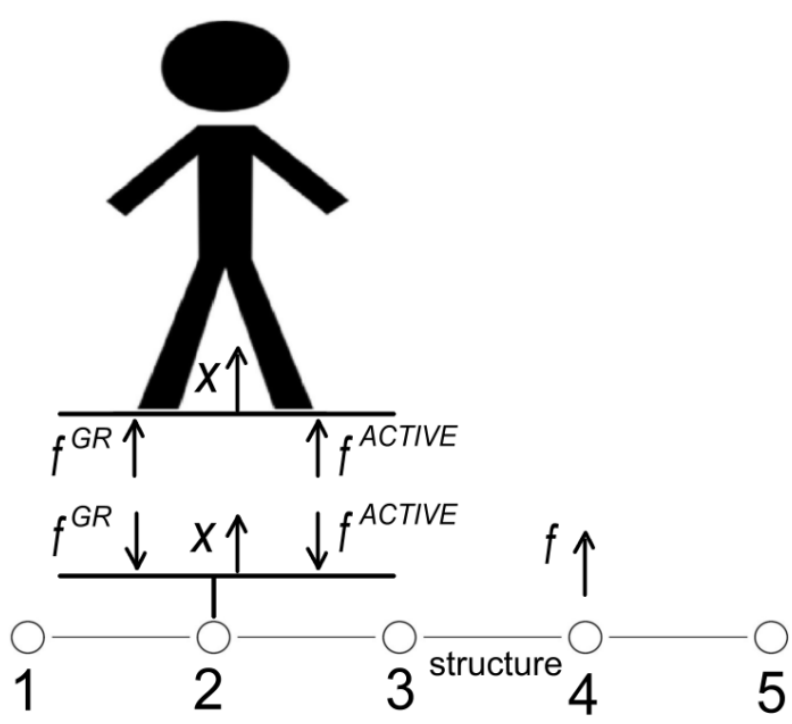

Figure 1: Human-structure interaction: PGRFs ( $\left.f^{G R}\right)$ and AGRFs (f ${ }^{A C T I V E}$ )

\subsection{The case of passive people}

In the case of passive people the AGRFs are null. As for the PGRFs, they can be described by the apparent mass of each person. The apparent mass $M^{*}(\omega)$ is the ratio between the force at the interconnection with the structure and the acceleration of the connection point $\ddot{x}_{i}$ :

$$
f_{i}^{\mathrm{GR}}(\omega)=M^{*}(\omega) \ddot{x}_{i}(\omega)=-M^{*}(\omega) \omega^{2} x_{i}(\omega)=H(\omega) x_{i}(\omega)
$$

If we consider more than one person, all the $f_{i}^{\mathrm{GR}}$ functions can be expressed in matrix form $\mathbf{H}$ and Equation 1 becomes:

$$
\left[\mathbf{G}^{-1}(\omega)+\mathbf{W H} \mathbf{W}^{\mathrm{T}}\right] \mathbf{x}(\omega)=\mathbf{G}_{\mathrm{H}}^{-1}(\omega) \mathbf{x}(\omega)=\mathbf{f}(\omega)
$$

Where $\mathbf{W}$ is a matrix representing the connection of $m$ subjects to the structure (i.e., it is a matrix composed by 0 and 1 elements [6]).

According to the Woodbury matrix identity [29], the new matrix $\mathbf{G}_{\mathbf{H}}$ of the FRFs of the new system composed by the structure and the people is:

$$
\mathbf{G}_{\mathbf{H}}=\left[\mathbf{G}^{-1}+\mathbf{W H} \mathbf{W}^{\mathrm{T}}\right]^{-1}=\mathbf{G}-\mathbf{G W}\left(\mathbf{H}^{-1}+\mathbf{W}^{\mathrm{T}} \mathbf{G} \mathbf{W}\right)^{-1} \mathbf{W}^{\mathrm{T}} \mathbf{G}
$$

To find the expression of $\mathbf{G}_{\mathbf{H}}$, the $H$ functions must be known for all the people. It is possible to use measured $H$ functions as well as approximated models available in literature [30][31].

\subsection{The case of active people}

In the case of active people the AGRFs are non-null. Equation 1 can be used to estimate the structural response due to people movement on the structure. To this purpose, we need an estimation of the AGRFs and PGRFs. 
As for the AGRFs, it is possible to build a database of forces for the considered kind of movement (e.g., as in [7] for people ascending and descending staircases). Figure 2 shows some examples of the collected AGRF time-histories for people ascending and descending staircases.
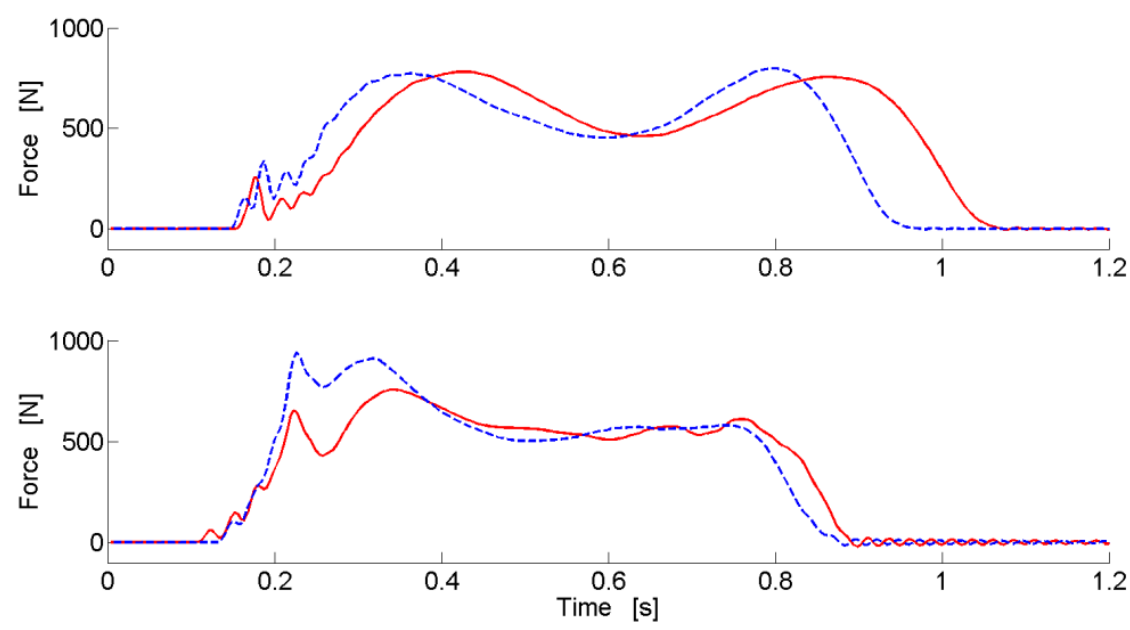

Figure 2: Two examples of recorded AGRF time-histories for people ascending a staircase (up) and two examples of recorded AGRF time-histories for people descending a staircase (down).

As for PGRFs, we can still use Equation 3, but we have to keep in mind that in this case the function $H$ changes with time because people change posture during the movement. Furthermore, people move over the structure so that the PGRFs change point of application in time. As for the former point, an equivalent apparent mass $M_{e q}^{*}$ is defined taking into account many different postures of the subject during the motion. Basically, each step is split into different positions ( $P$ positions) and the apparent mass $M_{a, i}^{*}$ is estimated (e.g., with experimental tests as in [7]) for each of these postures. Actually, each $M_{a, i}^{*}$ is the average of the apparent mass of different people in the given posture. Then, $M_{e q}^{*}$ is calculated as:

$$
M_{e q}^{*}(\omega)=\sum_{\mathrm{i}=1}^{P} \alpha_{i} M_{a, i}^{*}(\omega)
$$

where the $\alpha_{i}$ coefficients are the weights. They are chosen in order to describe properly the amount of time spent by the people in the corresponding posture within the cycle time $T$ (i.e., the time of a single step). In other words, we freeze $P$ postures within the movement cycle and we treat each of them as a static posture.

In this scenario, each person produces a PGRF related to the apparent mass $M_{e q}^{*}$. Then, the PGRFs can be applied to the structure in two different ways:

1. each PGRF (i.e., one for each person on the structure) is considered as a moving excitement. Hence, the equivalent matrix $\mathbf{G}_{\mathbf{H}}(\omega)$ (see Equation 4) changes in time;

2. a fraction of the apparent mass $m_{f r}^{*}(\omega)$ is calculated:

$$
m_{f r}^{*}(\omega)=\frac{m}{n_{d}} M_{e q}^{*}(\omega)
$$

Where $m$ is the number of people on the structure and $n_{d}$ is the number of points in which the structure is discretised. Then, $m_{f r}^{*}(\omega)$ is applied to each of the $n_{d}$ points. Thus, the PGRF in each point can be expressed as: 


$$
f_{i}^{\mathrm{GR}}(\omega)=m_{f r}^{*}(\omega) \ddot{x}_{i}(\omega)=-m_{f r}^{*}(\omega) \omega^{2} x_{i}(\omega)
$$

In terms of the full displacement vector $\mathbf{x}(\omega), \mathbf{f}^{\mathrm{GR}}$ becomes:

$$
\mathbf{f}^{\mathrm{GR}}(\omega)=\mathbf{W}_{\mathbf{n}} \mathbf{H} \mathbf{W}_{\mathbf{n}}^{\mathrm{T}} \mathbf{x}(\omega)=-\omega^{2} m_{f r}^{*}(\omega) \mathbf{W}_{\mathbf{n}} \mathbf{x}(\omega)
$$

where $\mathbf{W}_{\mathbf{n}}$ is a $n_{d} \times n_{d}$ identity matrix, $\mathbf{H}(\omega)$ is a $n_{d} \times n_{d}$ diagonal matrix containing the fractions of the equivalent apparent mass (i.e. $\mathbf{H}(\omega)=-\mathbf{W}_{\mathbf{n}} \omega^{2} m_{f r}^{*}(\omega)$ ). Substituting Equation 9 into Equation 1, we obtain (neglecting f):

$$
\left[\mathbf{G}^{-1}(\omega)+\omega^{2} m_{f r}^{*}(\omega) \mathbf{W}_{\mathbf{n}}\right] \mathbf{x}(\omega)=\mathbf{G}_{\mathrm{H}}^{-1}(\omega) \mathbf{x}(\omega)=-\mathbf{f}^{\mathrm{ACTIVE}}(\omega)
$$

where $\mathbf{G}_{\mathbf{H}}(\omega)$ is the $n_{d} \times n_{d}$ matrix representing the equivalent set of FRFs describing the dynamic behaviour of the joint system composed by the structure and the people. Obviously, the behaviour of this joint system is an average behaviour because $m_{f r}^{*}$ is employed.

The second approach of the previous list assumes a fixed form of $\mathbf{G}_{\mathbf{H}}(\omega)$ in time. Therefore, this assumption makes the simulation of the structure response fast and easy under the computational point of view. Moreover, when the number of people on the structure is increases, the accuracy of this easy-to-apply approach is expected to increase as well.

Finally, the response of the structure to the movement of people can be calculated as the convolution between the AGRFs and the unit impulse response functions of the joint system. These unit impulse response functions can be achieved by applying the inverse Fourier transform to the FRFs composing $\mathbf{G}_{\mathbf{H}}(\omega)$. As for the AGRFs to be applied, Figure 3 provides an example of how to build them by using the database of AGRFs mentioned previously. Such an example refers to a person ascending a staircase and the forces depicted are related to the force exerted by this person on three consequent steps.

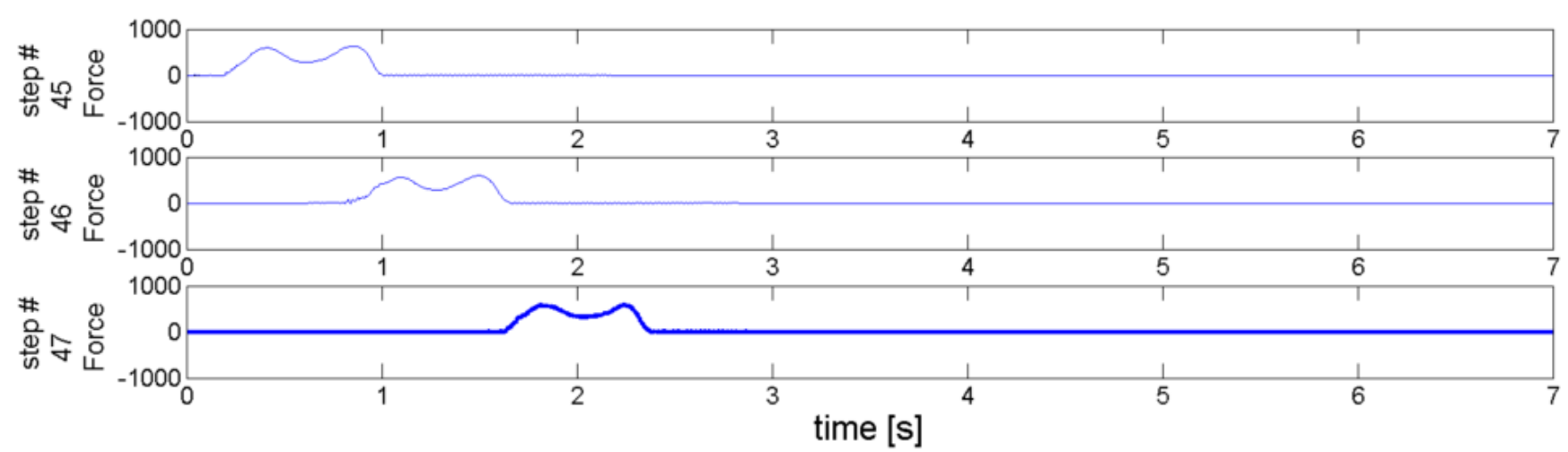

Figure 3: Simulation of the AGRFs exerted by a person ascending a staircase on three consequent steps. The portion of the timehistories where the forces are not null are recorded AGRFs like those of Figure 2. The force signals are in Newton.

\section{Experimental tests}

This section describes the experimental campaign carried out to validate the model presented in Section 2. Two staircases were used as test-structures. One, named S1, is made up of steel and marble, its length is $12.03 \mathrm{~m}$, its width is $1.80 \mathrm{~m}$ and the height is $5.22 \mathrm{~m}$. The second one, named S2, is made up of steel and has marble sheets on the steps, its length is 13.30 $\mathrm{m}$, its width is $1.80 \mathrm{~m}$ and the height is $5.60 \mathrm{~m}$. The modal parameters of the empty structures were identified by means of experimental modal analysis [32] and the values of the eigenfrequencies and non-dimensional damping ratios are gathered 
in Table 1 and Table 2. The modes taken into account are those in the frequency range $0-15 \mathrm{~Hz}$, which is the frequency band where the humans are mostly able to influence the dynamics of the structures.

\begin{tabular}{|c|c|c|}
\hline Mode number & $\omega_{j} /(2 \pi)[\mathrm{Hz}]$ & $\zeta_{j}[\%]$ \\
\hline 1 & 7.84 & 0.33 \\
\hline 2 & 8.89 & 0.43 \\
\hline
\end{tabular}

Table 1: Modal data identified experimentally for S1 in the frequency range 0-15 $\mathrm{Hz}$ (empty structure)

\begin{tabular}{|c|c|c|}
\hline Mode number & $\omega_{j} /(2 \pi)[\mathrm{Hz}]$ & $\zeta_{j}[\%]$ \\
\hline 1 & 6.70 & 0.33 \\
\hline 2 & 9.55 & 0.28 \\
\hline 3 & 10.75 & 0.29 \\
\hline 4 & 11.21 & 0.17 \\
\hline
\end{tabular}

Table 2: Modal data identified experimentally for S2 in the frequency range 0-15 $\mathrm{Hz}$ (empty structure)

\subsection{Tests with passive people}

This subsection presents some results related to the validation of the model of Section 2.1, developed to model the influence of passive people on the dynamic behaviour of the hosting structure. Different people were asked to stay in given points of the staircases. Then, the structure was excited by using an electro-dynamic shaker laid down on the staircase and moving a mass. Therefore, the excitement was provided employing the inertial effect (more details about the way to excite the structure can be found in [6][7]). Finally, the FRFs of the structure were estimated by measuring the structural response by means of accelerometers. Figure $4 \mathrm{a}$ and Figure $4 \mathrm{~b}$ show the FRFs (for some tests and points of the structure chosen as examples) in a given point of S1 and S2, respectively. The experimental results match the numerical expectations for both the structures, proving the reliability and accuracy of the proposed approach. Other tests gave a similar level of accordance between experiments and simulations. Therefore, they are not shown here for sake of conciseness.
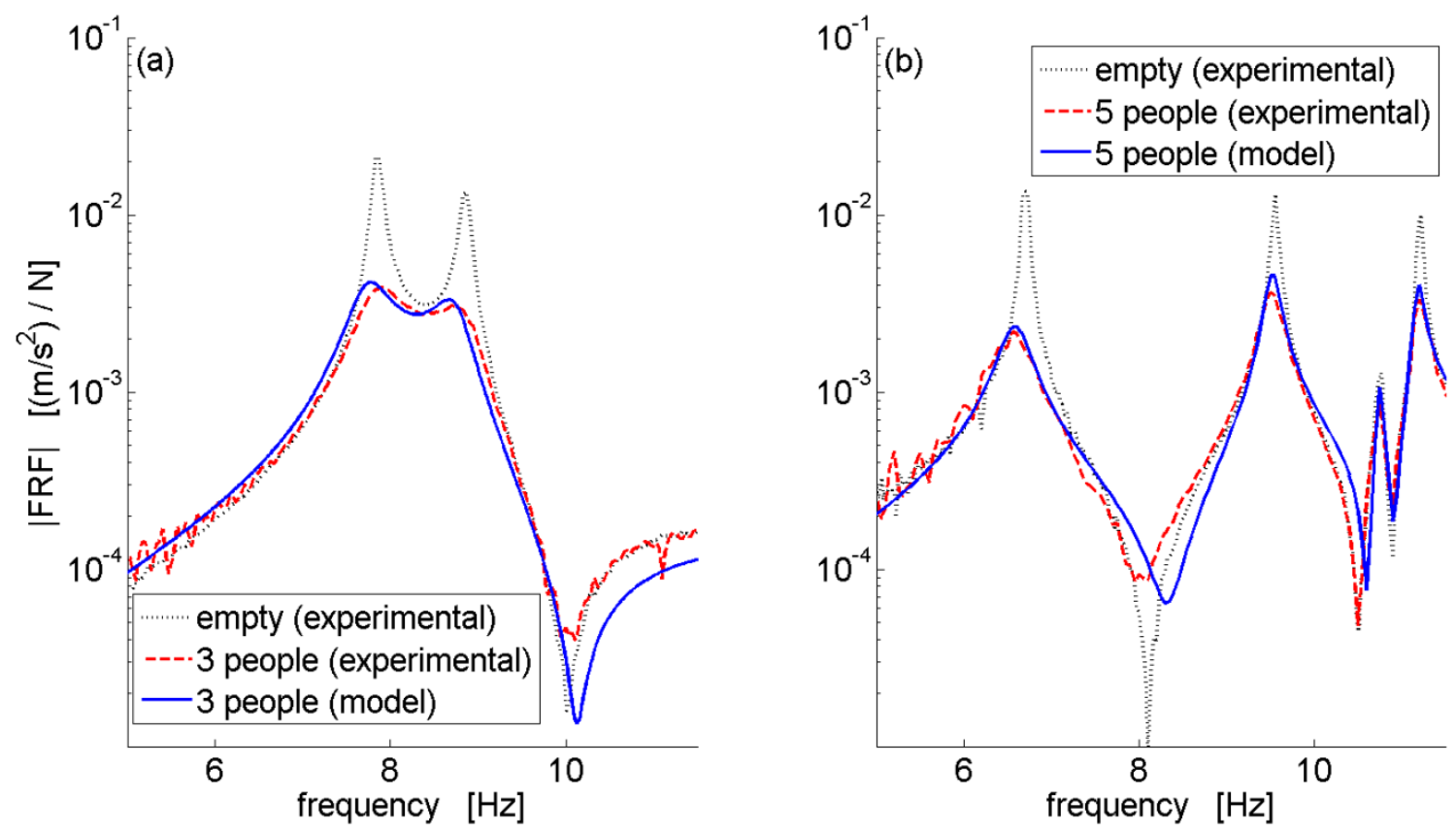

Figure 4: FRF for S1 (a) and S2 (b) 


\subsection{Tests with active people}

This subsection shows that the use of the model presented in Section 2.2 allows to improve the prediction of the structural response due to people moving. The tests were carried out by asking a given number of people to walk freely and all together on S1/S2. The acceleration responses of the structures were measured by means of accelerometers. The newly proposed approach is compared here to the experiments and to the use of the model of the empty structure directly excited by the AGRFs.

The mentioned comparison is carried out in terms of root mean square (RMS) of the time-responses. Here, the resulting RMS values are related to the point of maximum response for both S1 and S2. The RMSs are calculated in the frequency range $0-15 \mathrm{~Hz}$.

The RMS values coming from the use of the models is represented as a mean value plus/minus twice the standard deviation. Indeed, since the AGRFs are sampled from a database of AGRFs (see Section 2.2), the result of a single simulation depends upon the AGRFs extracted from the database. Therefore, each case (i.e., a given number of people walking on either S1 or S2) was simulated 100 times to achieve a statistical reliability of the results by building of the resulting RMS values distributions (which were found to have a Gaussian trend).

Figure $5 \mathrm{a}$ and Figure 5b show the results related to the tests on S1 with 3 and 9 people walking on the staircase, respectively. Figure $5 c$ and Figure $5 d$ show the results related to the tests on S2 with 5 and 10 people walking on the staircase, respectively. These plots demonstrate that the model proposed in Section 2.2 allows to satisfactorily estimate the experimental results. Furthermore, the use the FRF matrix $\mathbf{G}_{\mathbf{H}}$ as described in Section 2.2 allows to achieve results more accurate than the direct application of the AGRFs to the model of the empty structure (i.e., using $\mathbf{G}$ ).
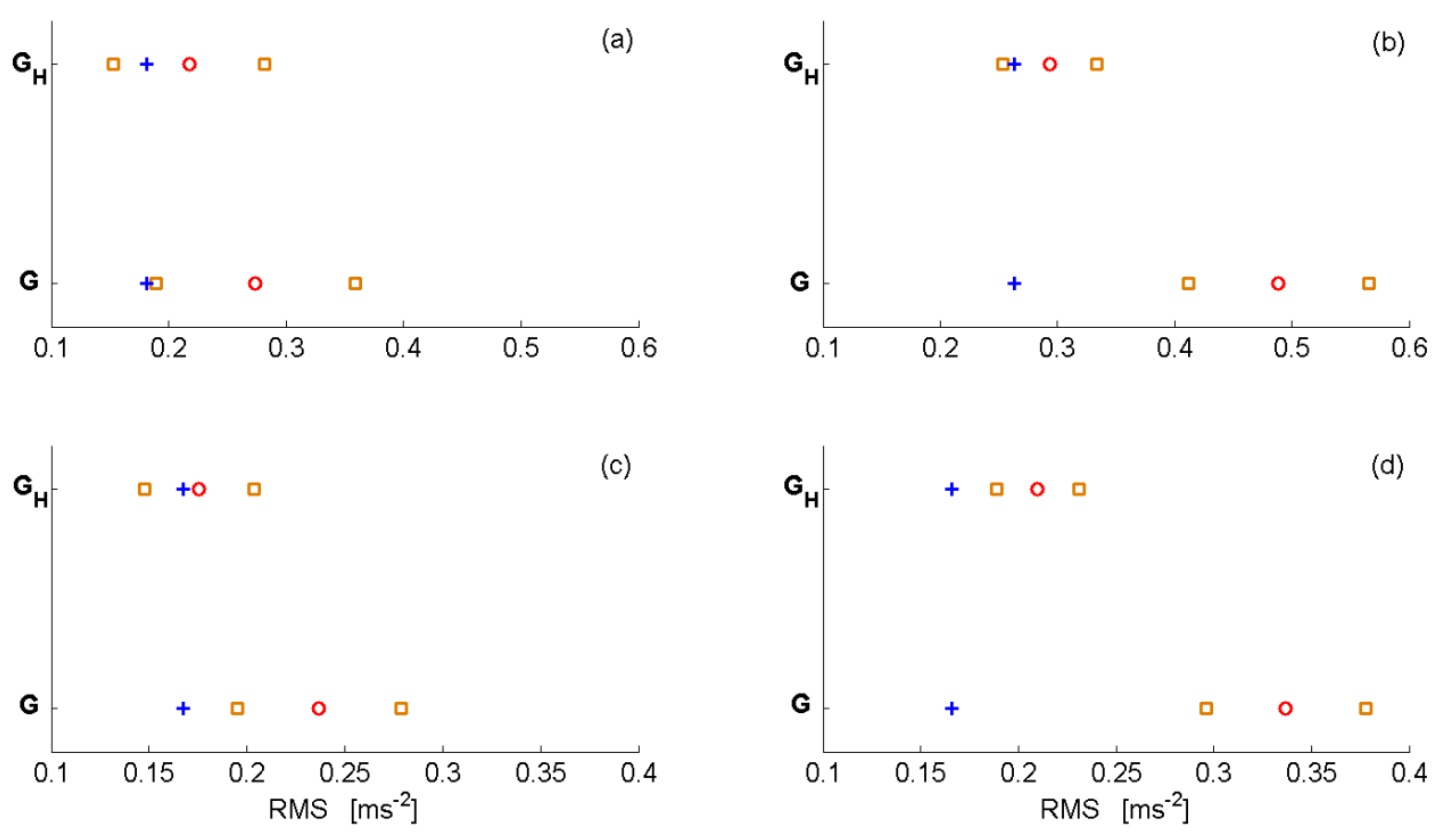

Figure 5: Resulting RMS values: S1 with 3 people walking (a), S1 with 9 people walking (b), S2 with 5 people walking (c) and S2 with 10 people walking (d). The symbol $G_{H}$ on the vertical axis refers to the results related to model presented in Section 2.2 , while the symbol

$G$ is related to the application of the AGRFs to the model of the empty structure. Plus symbols are related to experimental results, circle symbols are related to the mean value of the simulations results and square symbols are related to the mean value of the simulations results $+/$ - twice the standard deviation of the simulation results. 


\section{Conclusion}

This paper has dealt with a new approach to model the coupling between a slender structure and people occupying it. The proposed approach shows to be accurate and reliable when considering both passive and active people. Such a result is achieved by splitting the influence of each person as the sum of a passive ground reaction force and an active one.

The model has been validated by means of experimental tests performed on two different staircases showing a good agreement with experiments in terms of the predicted vibration levels and highlighting the fact that considering the empty structure response always leads to a consistent overestimation of the human induced vibrations.

\section{References}

[1] Sachse R, Pavic A and Reynolds P 2003 Human-structure dynamic interaction in civil engineering dynamics: a literature review Shock Vib. Dig. 35 3-18

[2] Živanović S, Pavic A and Reynolds P 2005 Vibration serviceability of footbridges under human-induced excitation: A literature review vol 279

[3] Racic V, Pavic A and Brownjohn J M W 2009 Experimental identification and analytical modelling of human walking forces: Literature review J. Sound Vib. 326 1-49

[4] Alexander N A 2006 Theoretical treatment of crowd-structure interaction dynamics Proc. Inst. Civ. Eng. - Struct. Build. 159 329-38

[5] Sim J, Blakeborough A and Williams M 2007 Modelling of joint crowd-structure system using equivalent reducedDOF system Shock Vib. 14 261-70

[6] Busca G, Cappellini a., Manzoni S, Tarabini M and Vanali M 2014 Quantification of changes in modal parameters due to the presence of passive people on a slender structure J. Sound Vib. 333 5641-52

[7] Cappellini A, Manzoni S, Vanali M and Cigada A 2016 Evaluation of the dynamic behaviour of steel staircases damped by the presence of people Eng. Struct. 115 165-78

[8] Venuti F, Bruno L and Bellomo N 2007 Crowd dynamics on a moving platform: Mathematical modelling and application to lively footbridges Math. Comput. Model. 45 252-69

[9] Venuti F, Racic V and Corbetta A 2016 Modelling framework for dynamic interaction between multiple pedestrians and vertical vibrations of footbridges J. Sound Vib. 379 245-63

[10] Van Nimmen K, Lombaert G, De Roeck G and Van den Broeck P 2014 Vibration serviceability of footbridges: Evaluation of the current codes of practice Eng. Struct. 59 448-61

[11] Figueiredo F P, da Silva J G S, de Lima L R O, Vellasco P C G da S and de Andrade S A L 2008 A parametric study of composite footbridges under pedestrian walking loads Eng. Struct. 30 605-15

[12] Mashaly E S, Ebrahim T M, Abou-Elfath H and Ebrahim O A 2013 Evaluating the vertical vibration response of footbridges using a response spectrum approach Alexandria Eng. J. 52 419-24

[13] Ingólfsson E T and Georgakis C T 2011 A stochastic load model for pedestrian-induced lateral forces on footbridges Eng. Struct. 33 3454-70

[14] Piccardo G and Tubino F 2012 Equivalent spectral model and maximum dynamic response for the serviceability analysis of footbridges Eng. Struct. 40 445-56 
[15] Toso M A, Gomes H M, Da Silva F T and Pimentel R L 2016 Experimentally fitted biodynamic models for pedestrianstructure interaction in walking situations Mech. Syst. Signal Process. 72-73 590-606

[16] Caprani C C and Ahmadi E 2016 Formulation of human-structure interaction system models for vertical vibration J. Sound Vib. 377 346-67

[17] Setareh M, Ph D and Asce M 2012 Vibrations due to Walking in a Long-Cantilevered Office Building Structure $J$. Perform. Constr. Facil. 26 255-70

[18] Sachse R, Pavic A and Reynolds P 2004 Parametric study of modal properties of damped two-degree-of-freedom crowd-structure dynamic systems J. Sound Vib. 274 461-80

[19] Reynolds P, Pavic A and Ibrahim Z 2004 Changes of modal properties of a stadium structure occupied by a crowd Proceedings of XXII International Modal Analysis Conference (IMAC) (Orlando (FL, USA))

[20] Cappellini A, Cattaneo A, Manzoni S, Scaccabarozzi M and Vanali M 2015 Effects of people occupancy on the modal properties of a stadium grandstand Proceedings of XXXIII International Modal Analysis Conference (IMAC) (Orlando (FL, USA))

[21] EN1990-Eurocode 2002 Basis of structural design

[22] Sètra 2006 Technical guide - assessment of vibrational behaviour of footbridges under pedestrian loading. Service d'Etudes techniques des routes et autoroutes

[23] ISO10137 2007 International Organization for Standardization - Bases for design of structures - serviceability of buildings and walkways against vibration

[24] Caprioli A, Reynolds P and Vanali M 2007 Evaluation of serviceability assessment measures for different stadia structures and different live concert events Proceedings of XXV International Modal Analysis Conference (IMAC) (Orlando (FL, USA))

[25] Caprioli A and Vanali M 2009 Comparison of different serviceability assessment measures for different events held in the G. Meazza stadium in Milano Proceedings of XXVII International Modal Analysis Conference (IMAC) (Orlando $(\mathrm{FL}, \mathrm{USA})$ )

[26] InstitutionofStructuralEngineers 2008 Dynamic performance requirements for permanent grandstands subject to crowd action: recommendations for management, design and assessment

[27] Ewins D J 2000 Modal testing: theory, practice and application (Baldock: Research studies press Ltd.)

[28] Brandt A 2011 Noise and vibration analysis - Signal analysis and experimental procedures (Wiley)

[29] Woodbury M A 1950 Inverting modified matrices (Princeton (NJ, USA))

[30] Matsumoto Y and Griffin M J 1998 Dynamic Response of the Standing Human Body Exposed To Vertical Vibration: Influence of Posture and Vibration Magnitude J. Sound Vib. 212 85-107

[31] Matsumoto $Y$ and Griffin M J 2003 Mathematical models for the apparent masses of standing subjects exposed to vertical whole-body vibration J. Sound Vib. 260 431-51

[32] Peeters B, Auweraer H Van Der, Guillaume P and Leuridan J 2004 The PolyMAX frequency-domain method : a new standard for modal parameter estimation? Shock Vib. 11 395-409 